\title{
LA ÉTICA MÉDICA EN DISCUSIÓN: ANÁLISIS DE LOS PRINCIPALES TEMAS SOBRE ÉTICA MÉDICA EN LA PRÁCTICA CLÍNICA TRATADOS EN REVISTAS NACIONALES DE MEDICINA Y BIOÉTICA 1998-2013
}

\author{
Paulina Ramos Vergara ${ }^{1}$, Paula Bedregal García ${ }^{2}$
}

Resumen: El objetivo de esta investigación es identificar los principales temas de la discusión sobre de ética médica en la práctica clínica en Chile que se suscitan en las revistas de Medicina y Bioética chilenas. Se identificaron 143 artículos que fueron sometidos a un análisis sistemático utilizando técnicas de comparación, codificación y análisis de textos propios del enfoque de la Teoría Fundamentada. Se distinguieron 30 temas en la discusión, en la que predomina el tratamiento de la ética desde la "deontología profesional". También se observan discursos emergentes de una ética que apela a bienes y valores superiores a los deberes profesionales en la búsqueda del bien del paciente, en el marco de un proceso de transformación del contexto en que se ejerce la práctica clínica.

Palabras clave: ética médica, práctica clínica

Medical ethics argumentation: analysis of main topics about medical ethics in clinical practice dealt in national journals of Medicine and Bioethics 1998-2013

\begin{abstract}
The aim of this investigation is to identify the main topics of the discussion about medical ethics in clinical practice in Chile. 143 articles in Chilean medical and bioethics journals were identified. They were put under a systematic study using comparison, codification and text analysis techniques of the Grounded Theory approach. 30 subjects were identified in the discussion, where the treatment of the ethics from a "professional deontology" predominates. Emergent speeches of an ethics that appeals to goods and values superiors to the professional duties in the search of the good of the patient can be found. The transformation of the context in which the clinical practice takes place is the background of the discussion.
\end{abstract}

Key words: medical ethics, clinical practice

A ética médica em discussão: análise dos principais temas de ética médica na prática clínica em revistas nacionais de medicina e bioética 1998-2013

Resumo: O objetivo desta pesquisa é identificar os principais tópicos da discussão sobre ética médica na prática clínica no Chile, presentes nas revistas nacionais de medicina e bioética. Identificaram-se 143 artigos que foram submetidos a uma análise sistemática, utilizando-se técnicas de comparação, codificação e análise de textos próprios da abordagem da Teoria Fundamentada. Identificaram-se 30 temas na discussão, nos quais predominam o tratamento da ética a partir da "deontologia profissional”. Também se observam discursos emergentes de uma ética que apela aos bens e valores superiores aos deveres profissionais na busca pelo bem do paciente, no âmbito de um processo de transformação do contexto em que é exercida a prática clínica.

Palavras-chave: ética médica, prática clínica

\footnotetext{
${ }^{1}$ Centro de Bioética, Pontificia Universidad Católica de Chile, Chile Correspondencia: pramosv@med.puc.cl

${ }^{2}$ Departamento de Salud Pública, Facultad de Medicina, Pontificia Universidad Católica de Chile. Centro de Bioética, Pontificia Universidad Católica de Chile, Chile
} 


\section{Introducción}

La reflexión ética sobre la práctica médica ha estado presente desde los orígenes de la profesión y ha acompañado su desarrollo a lo largo de la historia. Tradicionalmente, son los propios médicos quienes analizan y revisan los fundamentos ontológicos de su labor, cuestionan su quehacer profesional, formulan recomendaciones o establecen pautas normativas para regular sus actuaciones.

El desarrollo de la bioética y los nuevos desafíos que se plantean a la práctica clínica de la medicina hacen necesario un tratamiento más sistemático de la ética médica. Un modo de hacerlo es indagando en lo que escriben los médicos en las publicaciones referidas a la ética clínica, identificando los temas de que discuten y analizando los contenidos de la discusión. En el caso chileno, una exploración al respecto es posible a partir de las publicaciones nacionales de carácter científico de medicina y bioética.

Este estudio es una primera aproximación para conocer las voces en la discusión sobre ética médica en Chile, revisando para ello las publicaciones nacionales de Medicina y Bioética de los últimos quince años.

\section{Metodología}

La exploración de esta discusión se realizó en revistas chilenas indexadas a Scielo Chile, Lilacs y Medline que se encuentran bajo el título de "ciencias de la salud", a las que se puede acceder on line a texto completo y gratuito. También se analizó la Revista Vida Médica, publicación oficial del Colegio Médico de Chile AG, que, si bien no se encuentra indexada, es de relevancia en la discusión sobre ética médica en Chile.

La fecha considerada para iniciar la revisión de las revistas —año 1998 - responde a que este estudio comprende la discusión que ha tenido lugar en Chile a partir de la implementación de la denominada Reforma AUGE al sistema de salud, de nuevas políticas y normativas en salud, entre esas la Ley $\mathrm{N}^{\circ} 20.584$, de 13 abril del 2012, que regula los derechos y deberes de las personas en relación con las acciones vinculadas a su atención de salud.
En total se revisaron 2.224 números de 42 revistas nacionales de medicina y bioética. 41 de estas son reconocidas por su calidad científica, pertinencia, validez, originalidad y aporte, y calidad editorial, esto es, credibilidad. 25 pertenecen a sociedades médicas científicas de carácter nacional que cubren el espectro de la medicina clínica. Por su pertinencia directa al tema, se seleccionaron 143 artículos de 20 revistas. 11 son de las ya mencionadas sociedades médicas científicas y llevan el nombre de las mismas. Así, a modo de ejemplo, la Sociedad Chilena de Cirugía edita la Revista Chilena de Cirugia; la Sociedad Chilena de Neuropsiquiatría, la revista de su mismo nombre; la Sociedad Chilena de Pediatría, la Revista Chilena de Pediatría; la Revista Chilena de Radiología es de la Sociedad Chilena de Radiología; la Revista Chilena de Otorrinolaringología y Cirugía de Cabeza y Cuello de la respectiva sociedad del mismo nombre; y la Revista Médica de Chile, que es de la Sociedad Médica de Santiago. De los 143 artículos para analizar, 60 provienen de revistas de la Universidad de Chile y de la Pontificia Universidad Católica de Chile, también en forma importante de las sociedades médicas científicas.

La selección de artículos vinculados a la ética médica clínica se realizó con las siguientes palabras claves: ética, ética clínica. La revisión se realizó on-line y se complementó con la revisión manual de los textos. No se exploraron las dimensiones éticas de la formación del médico, del médico docente, del médico investigador, ni de su desempeño en salud pública.

Para identificar en los artículos los elementos de la discusión sobre ética médica se aplicó una metodología de análisis cualitativo, utilizando técnicas de comparación constante de la Teoría Fundamentada. Este método opera en forma inductiva, construyendo los elementos conceptuales a partir de la revisión, clasificación y comparación de las fuentes(1).

El análisis de los artículos se realizó, en primer lugar, con codificación abierta, que consiste en generar categorías nuevas a medida que se revisan los textos. Con este fin se marcaron párrafos y oraciones y se les asignó códigos. Ello permitió efectuar una descripción de los contenidos de los principales temas provenientes de los textos. A fin 
de generar una primera estructura de códigos que permitiera una comparación sistemática, se procedió a codificar una muestra inicial aleatoria del $10 \%$ de los artículos, resultando 16 códigos.

En segundo lugar, utilizando el sistema de comparación y análisis, se procedió a incorporar, modificar o eliminar códigos mediante el análisis de los artículos restantes, llegando a un total de 30 códigos. El análisis de códigos fue realizado por dos investigadores, uno de ellos experto en análisis cualitativo. El procesamiento de los datos fue apoyado usando el programa informático ATLAS.ti .

Esta investigación se realizó de acuerdo a las normas del Comité de Ética de Investigación de la Facultad de Medicina de la Pontificia Universidad Católica de Chile.

\section{Resultados}

Del análisis de los textos mediante codificación emergieron un total de 30 códigos, los cuales, en conjunto, dan cuenta de la discusión sobre ética médica en la práctica asistencial en revistas de medicina y bioética publicadas en Chile entre los años 1998-2013.

Los códigos se refieren a temas de diversa índole y alcance, algunos vinculados directamente a la práctica clínica del médico. Si bien este no se trata de un estudio cuantitativo, para efectos de considerar el orden de relevancia temática de cada uno de los códigos en el marco de los textos analizados, se tuvo en cuenta la cantidad de apariciones de cada uno de ellos. Con este dato, se procedió a clasificar los códigos en tres grupos. El primero de ellos determinó el código central de la discusión. El segundo, integrado por 6 códigos que, junto al central, son gravitantes en la discusión general sobre ética médica clínica. Y el tercero, que incluye 23 códigos que complementan y especifican la discusión (Tabla 1).
Tabla 1. Frecuencia de artículos en que se identifican los códigos en revistas chilenas (1998-2013)

\begin{tabular}{|c|c|}
\hline Código Central & \\
\hline Deberes y obligaciones del médico & 233 \\
\hline \multicolumn{2}{|l|}{ Códigos Secundarios } \\
\hline Relación médico-paciente & 107 \\
\hline Objeción de conciencia del médico & 80 \\
\hline Casos Clínicos Complejos & 78 \\
\hline Autonomía del paciente & 74 \\
\hline Ethos médico & 71 \\
\hline Prestación de Salud & 71 \\
\hline \multicolumn{2}{|l|}{ Códigos Complementarios } \\
\hline Profesionalidad & 65 \\
\hline Dimensión económica de la salud & 59 \\
\hline Actuación del médico según su conciencia & 53 \\
\hline Salud y cuidado del paciente & 53 \\
\hline Lex Artis & 48 \\
\hline Dimensión tecnológica de la salud & 46 \\
\hline Derechos del paciente & 44 \\
\hline Formación del médico & 40 \\
\hline Consentimiento del paciente & 38 \\
\hline Conflicto de intereses & 36 \\
\hline Sociedad democrática & 29 \\
\hline Dilema ético del médico & 27 \\
\hline Gestión clínica & 21 \\
\hline Conciencia moral & 19 \\
\hline Medicina Paliativa & 15 \\
\hline Responsabilidad del médico & 13 \\
\hline Estado y Políticas Públicas en Salud & 13 \\
\hline Ethos paternalista & 13 \\
\hline Rel médico - empresa farmacéutica & 11 \\
\hline Rel médico - establecimiento sanitario & 8 \\
\hline Relación médico - equipo sanitario & 6 \\
\hline Relación médico - sistema Isapre & 6 \\
\hline Investigación biomédica & 4 \\
\hline
\end{tabular}

Código central: deberes y obligaciones del médico

Los deberes y obligaciones del médico constituyen el código central del análisis de la discusión sobre ética médica de la práctica clínica, operando como eje articulador de otros temas y noción que incluye los demás códigos. 
Este código comprende múltiples referencias a normas de comportamiento dirigidas a los miembros de la profesión médica en sus relaciones y decisiones en la práctica clínica. De acuerdo con ello, el acto médico es calificado desde el punto de vista moral, con los términos "bueno" o "malo", "deseable" o "reprochable". Se trata de una persistente observación normativa de los actos que realiza el médico en el desempeño de su profesión, la cual se expresa principalmente por medio de referencias a obligaciones o deberes por cumplir, formuladas como imperativos: "los médicos deben...", "los médicos tienen que...". En los textos analizados, estas obligaciones emanan de distintas fuentes:

- Principios y valores, generales o propios del médico.

- La autonomía y derechos del paciente.

Práctica médica correcta (lex artis) y profesionalidad

Normativas y códigos de ética nacionales o internacionales, que incluyen varios de los elementos anteriores.

Se observa una referencia permanente a los deberes del médico, vinculados a los llamados principios de la bioética: beneficencia, no maleficencia, autonomía, justicia y equidad, los que aparecen siempre formulados como deberes y obligaciones del médico, ya sea en términos generales o que se desarrollen en aplicaciones a casos específicos. También como deberes y obligaciones se articulan los principios de la profesión médica, que remontan al juramento hipocrático y el origen de la medicina en occidente. Además, en los textos se registran referencias constantes a los "códigos de ética médica" de diverso origen, a declaraciones de asociaciones médicas, que expresan una lista de obligaciones que los médicos deben cumplir en su ejercicio profesional:

"Los médicos deben ser honestos con los pacientes y capacitarlos para que tomen decisiones informadas sobre su tratamiento".

"Los médicos deben trabajar activamente para eliminar toda discriminación en los cuidados de salud, sea por razones étnicas o raciales, de géne- ro, socioeconómicas, religiosas o de cualquier otra categoría social".

"Los médicos deben comprometerse a aprender toda la vida".

"Los médicos deben asegurarse de que los pacientes estén completa y honestamente informados antes de aceptar un tratamiento".

"Los médicos deben satisfacer el compromiso de confidencialidad".

"El médico debe trabajar con otros profesionales para reducir los errores, aumentar la seguridad de los pacientes, evitar el uso exagerado de los recursos y optimizar los cuidados de salud".

"Los médicos tienen el deber de resguardar los estándares científicos, promover la investigación y crear nuevos conocimientos, asegurando su apropiada utilización”.

"Los médicos tienen la obligación de reconocer, alertar al público en general y enfrentar los conflictos de intereses". (Artículo 93).

Las referencias a obligaciones del médico aparecen también a propósito del análisis de casos clínicos complejos y/o cuando se analiza el tratamiento de adultos mayores o los cuidados paliativos. También las referencias a las obligaciones aparecen con relación a situaciones en las que el médico ve condicionada su práctica clínica a otros intereses, principalmente de tipo económico.

Se observa que en el trasfondo general de las menciones a los deberes y obligaciones del médico aparece constantemente la relación médicopaciente.

\section{Relación médico-paciente}

Aparece en segundo lugar de las menciones en la discusión sobre ética médica. Los autores tratan este vínculo interpersonal como la piedra angular de la práctica médica y, por lo tanto, de la ética médica.

Las menciones a esta relación son principalmente "al deber ser" del vínculo que debe establecer el médico con el paciente. Al respecto, se registran 
numerosas referencias a la idea de que el bien del paciente se logra con un trato de respeto y deferencia.

De acuerdo con los hallazgos, los elementos que definen esta relación y a la vez constituyen la práctica médica son la vinculación intersubjetiva entre un médico y un paciente, fundada en el respeto y confianza recíproca, con fines terapéuticos, de carácter dialógico y comunicación continua, en la que hoy participa también la sociedad.

La discusión da cuenta de que el contexto político, económico y social está cambiando el tipo de relación médico-paciente. Los hallazgos consideran una transición del modelo "paternalista consentido", en que el médico ejerce un rol superior e incuestionable, dado su conocimiento, a un modelo de relación en que el paciente cobra protagonismo en las decisiones referentes a su salud, ejerciendo su autonomía. Algunos autores consideran los cambios como amenazas a la libertad profesional del médico y la instalación de una nueva asimetría de poder, en la que el médico queda ahora subordinado al paciente.

En la discusión se mencionan también riesgos de "desconfianza recíproca" e incluso de "deshumanización" de la relación médico-paciente, al limitarla a una prestación de servicios, con énfasis en la dimensión económica y contractual.

Esas amenazas pueden ser gravosas en la relación médico-paciente, de allí parece urgente "...reflexionar sobre el tema (relación médico-paciente), tanto más si la pureza del diálogo y las relaciones humanas pueden verse empañadas cuando por la interpretación puramente jurídica se entiende la prestación de un servicio como simple contrato entre partes" (Artículo 70).

\section{Objeción de conciencia del médico}

En los artículos analizados está presente también la objeción de conciencia del médico, entendida como su negativa, fundada en razones morales, a cumplir un cometido profesional exigido por una norma o autoridad.

En los textos se puede apreciar como en Chile la discusión sobre objeción de conciencia del médi- co se inicia a propósito de la esterilización voluntaria de hombres y mujeres y la anticoncepción en sus diferentes modalidades. No obstante, si bien la discusión sobre la procedencia de la objeción de conciencia del médico emerge a propósito de las políticas de salud reproductiva, también se extiende a otros temas.

"En el campo de la salud son múltiples los casos que propician el encuentro de la objeción de conciencia. Algunos de ellos son el aborto, la eutanasia, los procedimientos para desconectar a un enfermo declarado con muerte cerebral, entre otros. Así también en la oposición para atender un número exagerado de pacientes y en la resistencia a ser el brazo ejecutor de los pacientes terminales, a quienes les ha sido negada la mínima asistencia tanatológica y los cuidados paliativos humanizantes, como sucede además con la obstinación terapéutica en procedimientos fútiles" (Artículo 33).

La objeción de conciencia del médico aparece como un acto individual fundado en el respeto de los valores o conciencia personal. El objetor no busca cambiar las normas institucionales o sociales. En este punto, se encuentran nuevamente indicaciones al médico sobre cómo debe actuar en relación con el paciente.

Sin embargo, algunos autores miran con recelo la objeción de conciencia del médico en cuanto afecta el derecho de los pacientes, "dejándolos ausentes de la resolución del conflicto y como si ello fuera sólo un accidente" (Artículo 25). En esta línea, la objeción de conciencia del médico constituiría una falta del deber profesional, al negar una prestación de salud que por derecho reclama el paciente. "Se ha afirmado incluso que un médico objetor no debería ser médico; que los médicos objetores deberían confinarse a instituciones de salud que respalden su actuar o a especialidades donde no se expongan a situaciones clínicas que objetar" (Artículo 130).

Otros autores estiman que el derecho a rechazar realizar determinadas prestaciones que contrarían sus valores debería extenderse a las instituciones prestadoras de salud en cuanto agentes morales. "El asunto se complica más aún por la presencia de instituciones médicas de excelencia que tienen orientación doctrinaria cuyos valores a respetar 
pueden diferir de lo establecido como norma en la esfera pública, produciendo diversos flancos polémicos" (Artículo 57).

El tratamiento de la objeción de conciencia del médico en estos textos da cuenta de la tensión que ocasiona en el ámbito social, en la institucionalidad y en el establecimiento sanitario.

\section{Casos clinicos complejos}

Otro tema recurrente de la discusión sobre ética médica clínica corresponde al análisis de los casos clínicos complejos. La mayor parte de estos artículos discurren sobre lo que denominan "consideraciones éticas" o "implicancias éticas" de un caso clínico determinado, prescribiendo recomendaciones para futuras actuaciones de los médicos, tanto respecto de la conveniencia de aplicación de un determinado tratamiento, como de las pautas que deben aplicarse en la relación con el paciente. Los temas que registran más análisis en tanto casos complejos son:

- Anticoncepción de emergencia.

- Aborto terapéutico y aborto en general.

- Trastornos graves en el embarazo (anencefalia, embarazo tubario).

- Técnicas de reproducción asistida.

- Transfusiones de sangre en Testigos de Jehová.

- Cuidados paliativos.

- Tratamiento de adultos mayores.

- Maltrato infantil.

En este contexto, los comités de ética clínica cobran un rol más relevante como asesores de los médicos en el análisis y toma de decisiones en el contexto de la relación clínica (Artículo 31).

\section{Autonomía del paciente}

Otro tema relevante en los textos analizados es el reconocimiento por el médico de la capacidad de autodeterminación del paciente en lo que respecta a su salud y tratamientos correspondientes. Los autores enfatizan el deber del médico de realizar su quehacer respetando las convicciones, aspiraciones, deseos y derechos no codificados del paciente. "El principio hipocrático de beneficencia ha de complementarse con la noción que el paciente tiene de su propio bien" (Artículo 94).

La discusión también considera la determinación del bien del paciente y la toma de decisiones respecto de este: "El paciente es el único que puede informar a su tratante acerca de lo bueno, adecuado y lo que está dispuesto a aceptar como tratamiento. Es aquí donde toma sentido el principio de respeto de la autonomía del paciente, que no debe situar al médico solo como el ejecutante técnico de los deseos del consultante, sino que debe entenderse como la única manera que el médico tiene para conocer lo que quiere y a lo que está dispuesto a someterse el sujeto a quien atiende" (Artículo 13).

Para el ejercicio de la autonomía del paciente es condición indispensable el deber del médico de entregarle en forma oportuna y clara la información correspondiente y constatar que el paciente la comprendió y está en condición de suscribir el "consentimiento informado", figura presente cotidianamente en la práctica médica, según estos textos.

En los casos de pacientes con capacidad limitada — por edad, salud o nivel sociocultural_-, el médico está obligado, por el respeto a esa autonomía limitada, a ejercer un rol más activo que le permita conocer y responder a los reales requerimientos de su paciente.

En la discusión además aparece la necesidad de diseñar protocolos que permitan estandarizar los procedimientos para evaluar la capacidad de los pacientes para la toma de decisiones, a fin de evitar la vulneración de su derecho a la autodeterminación.

El discurso predominante apunta a reconocer la obligación del médico de respetar la voluntad de su paciente, incluso por sobre lo que indiquen otros intereses y actores tan relevantes como su familia. También son los médicos los que se comprometen a establecer relaciones de confianza con su paciente sobre todo ante situaciones de sufrimiento y cercanía de la muerte. Además 
prescriben que en algunos casos es necesario priorizar el alivio del dolor, precisamente para permitir el ejercicio de la autonomía sin coacciones. Por último estiman que, particularmente para los médicos, los cuidados paliativos constituye un campo relevante para el respeto de la autonomía del paciente.

\section{Ethos médico}

En los textos analizados, Ethos médico refiere a la forma de ser, a las pautas de comportamiento de los médicos en su práctica clínica. También considera percepciones y valoraciones del médico respecto de su propio estatus y rol.

El Ethos médico, en cuanto a lo que el médico es, apunta a descripciones del ejercicio profesional en el tiempo y de los efectos de los cambios del entorno en su práctica. El Ethos médico, en cuanto al "deber ser", si bien no siempre en términos explícitos, refiere a la dimensión ética del ejercicio de la profesión, al bien que se persigue, a los valores en juego, a la libertad.

En la discusión se consigna que en los últimos decenios la sociedad desacralizó la figura y el rol de los que gozaba el médico desde tiempos inmemoriales. El cambio del estatus social del médico impacta directamente el ejercicio médico clínico: "El médico y el ejercicio de su profesión fueron expulsados irremisiblemente de la casa del sacerdote. El ethos del médico emigró desde el carácter sacerdotal que lo investía hacia otro que se parece en mucho al de un "asesor", que sopla al oído qué es lo mejor que se podría hacer en una determinada situación, y que luego acompańa en una decisión que el paciente mismo y no él ha tomado" (Artículo 105).

En cuanto al "deber ser" del médico, las referencias consideran el modo en que debe comportarse, los valores superiores que debe respetar en el ejercicio de su profesión, más allá de derechos y obligaciones.

En ambas acepciones del Ethos médico, la discusión considera que los cambios han afectado la identidad e imagen del médico y requieren adaptarse a nuevos requerimientos: "Los médicos, a su vez, sufren en su dignidad laboral y personal, ya que se ven obligados al ejercicio de una medicina que pone en tensión sus ideales profesionales y personales más preciados. Así, en ocasiones tienen que adaptarse a las exigencias e intereses de las instituciones de salud y a ejercer una medicina a la defensiva" (Artículo 47).

En la discusión aparece, entre las formas de responder a los nuevos desafíos, redefinir lo que debe ser un buen médico y las relaciones en la práctica médica en el contexto actual. Y precisamente considerar la profundidad y extensión de la dimensión ética del ejercicio de la medicina y de la relación médico-paciente.

\section{Prestación de salud}

En los textos analizados emerge otro objeto problemático que impacta en la práctica clínica. Se trata de una concepción determinada de la acción del médico, circunscrita a su carácter técnico, contractual y estandarizado. El énfasis del carácter contractual de la relación terapéutica: limitar el acto del médico a una prestación de salud, a un servicio profesional específico a cambio de un pago en el que intervienen terceros.

Esta transformación del sentido del acto médico lleva consigo la incorporación de la valorización económica específica de las actuaciones de los médicos. También considera la segmentación y clasificación de los procedimientos por criterios médicos, de política pública y de gestión económica de la salud. De esta forma, las atenciones de salud quedan tipificadas como prestaciones específicas, que pueden otorgar determinados especialistas y dentro de los tiempos asignados en la atención.

En los textos se observa un rechazo a limitar el ejercicio asistencial a una prestación, en cuanto amenaza la profesión y eventualmente terminará desnaturalizándola. La relación médico-paciente, entendida como prestación, está ahora mediada por terceros. Esto es calificado también como problemático.

Con todo, algunos doctores —en primera persona- consideran posible y deseable renovar la reflexión ética y los fundamentos mismos de la medicina: "Los doctores toman partido: los nostálgicos que sólo añoran un glorioso pasado y 
condenan el presente, y los menos, que pensamos que es nuestra obligación repensar nuestra actividad profesional, proponiendo cambiar el paradigma clásico del ejercicio de la medicina. Y en este enfoque hay dos aspectos: el problema de la doble lealtad por la presencia de terceros pagadores y cómo los conflictos de intereses que de ello surgen debilitan el modelo hipocrático al enfrentarlo a la ética corporativa; por otra parte, la necesidad de que el médico asuma su responsabilidad en la distribución de recursos para conservar su autonomía. Y si no somos nosotros mismos, ¿quiénes tomarán el liderazgo para que los principios de la profesión no se vean aún más afectados?" (Artículo 95).

\section{Relación entre código central y secundarios}

Como se ha señalado, el código central de la discusión sobre ética médica es la referencia a deberes y obligaciones del médico. Puesto en relación con los demás códigos que emergen de la discusión, se observan un conjunto de vínculos directos e indirectos. La figura 1 muestra un esquema general, ilustrando los vínculos entre el código central y los secundarios. Las flechas indican que los temas están conectados, es decir, que aparecen vinculados en los textos analizados. La dirección de las flechas señala que un tema impacta en el tratamiento del otro al cual se dirige, como es el caso de la objeción de conciencia y los casos clínicos complejos. Entre algunos temas se observan relaciones bidireccionales.

\section{Relaciones entre Categoría Central y Secundarias}

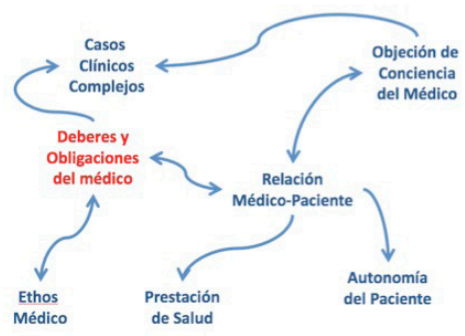

Figura 1: Organización general de la discusión sobre ética médica en Chile. Código central y códigos secundarios
La relación médico-paciente constituye el centro de la práctica clínica y, por ello, está siempre presente en la discusión sobre ética médica clínica. De esta forma, los contenidos específicos de los deberes y obligaciones del médico que aparecen en los textos analizados, están casi por completo referidos a la relación con el paciente. Es el médico el responsable de generar y mantener esta relación en todas sus dimensiones: buen trato, construcción de la confianza, comunicación y escucha, entrega de información y asegurar su comprensión.

Al mismo tiempo, en los textos se puede observar cómo la transformación de la relación médicopaciente exige de los médicos nuevas obligaciones y deberes, entre estos, el respeto por la autonomía del paciente, el cumplimiento de los derechos del paciente y la aplicación del consentimiento informado.

Se produce entonces un doble movimiento que alimenta la ética médica de nuevos contenidos y desafíos vinculados a la transformación de la relación médico-paciente.

En la discusión sobre ética médica es relevante la relación entre el código central y la referencia a casos clínicos complejos. Un importante número de los artículos refiere a aspectos éticos de casos clínicos considerados especialmente complejos, ya sea porque corresponden a situaciones límite entre la vida y la muerte, al enfrentamiento entre dos o más valores o al principio del doble efecto de la acción de médico. Esta relación muestra cómo uno de los ejes del discurso sobre ética médica está conformado por la derivación de deberes y obligaciones del médico ante casos clínicos éticos especiales. El mismo esquema de argumentación se repite en casi todos los artículos que abordan este asunto:

- Se presentan las características y relevancia del caso dentro de la práctica clínica.

- Se revisan los elementos éticos en juego presentes en el caso.

- Se realiza un análisis en el que el autor toma una postura determinada. 
- Se formulan pautas de actuación que deben seguir los médicos ante esos casos.

Entre los deberes del médico y el Ethos médico también se observa un doble movimiento. Por una parte, una de las formas en que se expresa el Ethos médico es a través de la formulación de deberes y obligaciones que emanan de principios y valores. En esta línea se inscriben los llamados a recuperar la imagen y dignidad de la profesión médica, deteriorada por los conflictos de interés y la presión de los aspectos tecnológicos y económicos de la salud. Por otra parte, la revisión o reafirmación de deberes y obligaciones del médico afectan el comportamiento del médico y su imagen. El Ethos médico, como forma de ser y como imagen proyectada, se va modificando con los cambios en el contexto del ejercicio de la profesión médica. Dentro de esta variante se encuentran las referencias a la transición desde un ethos paternalista tradicional a un ethos profesionalizante.

Por su parte, la objeción de conciencia del médico es revisada en los textos con relación a la discusión de casos clínicos complejos, tales como "eutanasia" o solicitudes de pacientes que pueden cuestionar la libertad del médico en su más amplia dimensión — como es el caso de los Testigos de Jehová-, o de la esterilización de una persona con discapacidad mental. La negativa a actuar del médico surge precisamente a propósito de situaciones en las que concepciones valóricas o determinados principios se ven puestos en riesgo por las intervenciones solicitadas por el paciente. Además, la posibilidad de la objeción de conciencia del médico surge por su factibilidad de actuar también con los recursos farmacológicos y tecnológicos a su alcance.

La objeción de conciencia del médico y la relación con el paciente son conceptos que se vinculan en una doble dirección. El origen de la situación es creada por la solicitud o intención del paciente que genera conflictos con los valores del médico: "Si bien la objeción de conciencia se puede dar en el nivel médico-Estado o médico-institución de salud, es en la relación médico-paciente en la que se produce el conflicto moral más profundo. Es ahí donde se enfrentan, eventualmente, la autonomía del médico con la del paciente" (Artículo 130).
De esta forma, se defiende también la autonomía del médico en su relación con el paciente: "La autonomía del paciente merece respeto, sin lugar a dudas, pero no por eso se puede mermar a su vez la autonomía e integridad del profesional" (Artículo 130).

Por otro lado, hay quienes defienden los derechos y la autonomía del paciente por sobre la objeción de conciencia: "La utilización de un lenguaje normativo con alto poder de persuasión moral detrás de los derechos humanos, invocando la objeción de conciencia para no realizar ciertos deberes profesionales, esconde una parte significativa de esta tensión. Los derechos entre profesionales y usuarios se enfrentan, dejando a estos últimos ausentes de la resolución del conflicto y como si ello fuera solo un accidente. Los usuarios del sistema de salud deben ser protegidos en sus derechos. Se debe buscar fórmulas transparentes, intentando maximizar ambos actores y evitando la suma cero. Pero, ante el límite y sin otra opción, los prestadores de salud deberían hacer honor a sus responsabilidades funcionarias y profesionales" (Artículo 25).

La aplicación de la objeción de conciencia conlleva también, según los textos, deberes del médico para con su paciente, sobre todo en relación a la explicación clara de su razones de objetor, la derivación a otros profesionales y el posterior tratamiento del paciente después del procedimiento aplicado.

En la discusión sobre la relación terapéutica cobra protagonismo "la autonomía del paciente". La irrupción de la autonomía del paciente fue inobjetable luego de los escándalos en la experimentación médica, especialmente en la Alemania nazi, dando nacimiento al Código de Nuremberg. El movimiento de emancipación de los pacientes ha logrado consolidar el consentimiento informado, no solo en su forma de derecho propio del sujeto, sino también como extensión en la representación, incluso más allá de la condición de incapacidad, mediante los modelos de testamentos vitales o directrices previas. La relación médico-paciente, entendida como interacción de dos sujetos autónomos, se sustenta en el reconocimiento del paciente como un interlocutor válido en un proceso de diálogo. 
La calificación del acto del médico como una prestación de salud despersonaliza la relación médicopaciente, predominando aspectos contractuales y económicos: "También vemos, en el último tiempo, la implementación de medidas tendientes a reducir las llamadas 'listas de espera' de enfermos con patologías susceptibles de soluciones quirúrgicas. Se contrata para ello a médicos que viajan especialmente a realizar actos quirúrgicos a pacientes que conocen recién en el pabellón de cirugía y a los que no verán más después de terminada la operación. Estos médicos actúan solo como ejecutores de una técnica quirúrgica. A pesar de la corrección de lo dañado, la insatisfacción de esos sujetos será inevitable. Nada impedirá que cada uno de ellos sienta haber sido tratado como una máquina a la que se le cambió una parte descompuesta" (Artículo 10).

En este sentido, la organización de la salud en prestaciones estandarizadas termina modificando el comportamiento del médico.

\section{Discusión}

La noción de "deberes y obligaciones" es el tema central de la discusión sobre ética médica en la práctica clínica, enfatizando el deber ser, derivado de sus códigos deontológicos, impregnando los demás temas; entre ellos, la relación médicopaciente, la objeción de conciencia del médico, el abordaje de casos clínicos complejos, la autonomía del paciente, el Ethos médico y la atención de salud entendida como prestación contractual.

En la gran mayoría de los artículos revisados los autores reflexionan desde su experiencia sobre temas de su práctica clínica, los valores en juego propios de su profesión, plantean su pensamiento y sugerencias y, en algunos casos, recurren a otros autores. Por lo general, los autores no explicitan los marcos antropológicos ni filosóficos desde los que escriben, sino que se trata de reflexiones personales que en algunos casos consideran los deberes de sus códigos profesionales, y en otros un análisis que considera los cuatro principios de Georgetown: autonomía, beneficencia, no maleficencia, justicia.

Dentro de los artículos revisados, la investigación empírica en este tema es escasa. Los artículos ba- sados en estudios empíricos refieren principalmente a las percepciones de los pacientes y de estudiantes de medicina. No se detectaron estudios que indaguen en las prácticas médicas o en las percepciones de los propios médicos sobre el tema. Con todo, es necesario señalar que una de las limitaciones de este análisis es que la muestra hemerográfica no incluye revistas filosóficas, jurídicas, ni de ciencias sociales que traten la ética médica clínica.

No obstante, este primer análisis permite señalar un proceso de revalorización de la ética como fundamento del actuar profesional. De allí que varios autores plantean la necesidad prioritaria de educar el juicio moral de los futuros médicos, en una comunidad universitaria en que los valores se vivan, motiven y desarrollen. Según los autores, el nuevo contexto del ejercicio de la medicina demanda la configuración de nueva alianza terapéutica, como asimismo configurar las garantías y límites de la libertad del paciente y del médico.

Una primera conclusión de este estudio es que se requiere un trabajo sistemático y riguroso de ordenamiento conceptual de los elementos que emergen de la discusión, integrando el estado internacional de la cuestión en la materia, con el propósito de producir una estructura analítica completa de las temáticas relativas a la ética médica de la práctica clínica.

Otra conclusión que se deriva de este estudio es la necesidad de realizar investigaciones de carácter empírico sobre la ética del médico en la práctica clínica, estudiando los discursos, las percepciones y las prácticas de los profesionales. La lista de temas identificados como parte de la discusión y sus relaciones constituye un insumo adecuado para generar una agenda temática que oriente la investigación futura en el área.

La agenda de nuevos estudios debiera abordar aquellos elementos del contexto del ejercicio de la profesión médica que han sufrido transformaciones en los últimos años, y realizar investigaciones acerca de la relación médico-paciente y de los desafíos éticos que se enfrentan desde el ejercicio de la profesión ante:

- El desarrollo de la industria y del mercado de 
la salud en Chile.

- La implementación de la reforma de salud.

- La relación del médico con el establecimiento sanitario.

- La interacción de médico con otros profesionales de la salud.
- La relación del médico con la industria farmacéutica.

La claridad conceptual y la evidencia empírica son tareas complementarias necesarias para enfrentar la complejidad de los problemas éticos de la práctica asistencial del médico.

\section{Referencias}

1. Glaser BG, Strauss AL. The discovery of grounded theory: strategies for qualitative research. New York: Aldine Publishing Company; 1967.

Recibido: 12 de julio de 2015

Aceptado: 20 de octubre de 2015 